\title{
Global Dynamics of Some Periodically Forced, Monotone Difference Equations
}

\author{
J. M. CUSHING \\ Department of Mathematics \\ University of Arizona \\ Tucson, AZ 85721-0089 \\ SHANDELLE M. HENSON \\ Department of Mathematics \\ The College of William and Mary \\ Williamsburg, VA 23187-8795
}

J. Difference Equations and Applications 7 (2001), 859-872

Dedicated to C. D. Ahlbrandt on the occasion of his sixtieth birthday

\begin{abstract}
We study a class of periodically forced, monotone difference equations motivated by applications from population dynamics. We give conditions under which there exists a globally attracting cycle and conditions under which the attracting cycle is attenuant.

Keywords: difference equations, periodic forcing, global attractors, periodic cycles, cycle average

AMS Classification codes: 39A11, 92D25
\end{abstract}

\section{Introduction}

If $g(y)$ is an increasing function of $y$, the solution sequences of the difference equation

$$
y_{t+1}=g\left(y_{t}\right), \quad t=0,1,2, \cdots
$$

are monotonic and hence, if bounded, converge. Assuming $g(y)$ is continuous and letting $t \rightarrow \infty$ in (1) we find that the limit $y_{e}=\lim _{t \rightarrow \infty} y_{t}$ of a convergent solution is necessarily an equilibrium solution $y_{t} \equiv y_{e}$, since $y_{e}$ is a root of the equilibrium equation $y=g(y)$. We are motivated by difference equations of this monotone type that appear as applications in population dynamics.

An example is the Beverton-Holt equation

$$
y_{t+1}=r \frac{1}{1+c y_{t}} y_{t}, \quad y_{0}>0
$$


where $r$ and $c$ are positive constants. If $r \leq 1$, the solution of this initial value problem converges to the equilibrium $y_{e}=0$. If $r>1$ the solution converges to the positive equilibrium $y_{e}=K=\frac{r-1}{c}$. With $K$ introduced explicitly, the Beverton-Holt equation becomes

$$
y_{t+1}=r \frac{1}{1+(r-1) \frac{y_{t}}{K}} y_{t} .
$$

Because all solutions are monotonic and converge, this difference equation is, from a dynamics point of view, a more appropriate analog to the famous logistic differential equation than are non-monotone maps such as the so-called "discrete logistic" equation $y_{t+1}=r\left(1-c y_{t}\right) y_{t}$ (with $\left.K=\frac{r-1}{r c}\right)$ or the Ricker equation $y_{t+1}=r e^{-c y_{t}} y_{t}$ (with $K=\frac{1}{c} \ln r$ ). As is well known, such "one hump" maps can have periodic solutions and even chaotic solutions.

In theoretical ecology, the parameters $r$ and $K$ play an important role. The coefficient $r$ is considered a characteristic of the population (its "inherent growth rate"), determined by life cycle and demographic properties such as birth rates, survivorship rates, etc. The coefficient $K$ is considered a characteristic of the habitat or environment (called the "carrying capacity"), e.g., resource availability, temperature, humidity, etc. The autonomous equations and their equilibrium theory above are appropriate in biological applications only if the $r$ and $K$ are constant over time. In cases where one or both of these parameters fluctuate in time (which is, of course, quite common for biological populations), the model equations become non-autonomous. For example, periodic fluctuations are common (caused, for example, by annual or daily fluctuations in the physical environment), in which case $r$ and/or $K$ become periodic functions of time. While periodic differential equation population models have been considered in the literature, relatively little attention has been paid to periodic difference equation population models. (See, however, [3], [5], [6], [7], [8], [9].)

One ecological question that has been studied by means of periodic models concerns the effect of a periodic environment on a population, i.e., the effect of a periodic $K$. Attention has focused on whether or not a population is adversely affected by a periodic environment (relative to a constant environment of the same average carrying capacity). Early results based on the logistic differential equation implied that a periodic carrying capacity $K$ is deleterious in the sense that the average of the resulting population oscillations is less than the average of $K$ [1], [2], [10], [11]. Later results showed this assertion can be model dependent [4], [12]. A recent study utilizing non-monotone difference equations has demonstrated the latter point. In [3], [9] it is shown, by mathematical analysis and laboratory experiments, that it is possible for a periodic environment to be advantageous for a population in the sense that average densities are greater in a periodic environment than in a constant environment.

In this paper, we show that a periodic environment is always deleterious for populations modeled by a class of monotone difference equations. This result is analogous to that in [1], [10] for the logistic differential equation.

In a periodic environment version of the Beverton-Holt equation we replace $K$ by a periodic sequence $K=K(t)$. We will restrict our attention in this paper 
to oscillations of period two. Therefore, we take $K=K_{a v}\left(1+\alpha(-1)^{t}\right)$ where $K_{a v}$ is the average of $K$ over time and $\alpha \in[0,1)$ the relative amplitude of the oscillation. (We have arbitrarily chosen the oscillation so that its maximum occurs at even time units. Our results remain valid if instead the maximum occurs at odd time units, i.e., $\alpha \in(-1,0]$.) In the resulting equation, we can eliminate the parameter $K_{a v}$ by the rescaling $x_{t}=y_{t} / K_{a v}$ and obtain the equation

$$
x_{t+1}=r \frac{1}{1+(r-1) \frac{x_{t}}{1+\alpha(-1)^{t}}} x_{t} .
$$

Motivated by the periodically forced Beverton-Holt equation (2) we consider a general class of periodically forced equations of the form

$$
x_{t+1}=f\left(\frac{x_{t}}{1+\alpha(-1)^{t}}\right) x_{t}, \quad \alpha \in[0,1)
$$

where the function

$$
h(x) \stackrel{\circ}{=}(x) x
$$

satisfies the conditions

$$
\begin{array}{ll}
\mathrm{A} 1: & h \in C^{0}\left(R^{+}, R^{+}\right) \cap C^{2}\left(R_{0}^{+}, R^{+}\right) \\
& h^{\prime}(x)>0, h^{\prime \prime}(x)<0 \text { for all } x \in R_{0}^{+} \\
& h(0)=0, h(\infty)<\infty
\end{array}
$$

Here $R^{+}=[0, \infty), R_{0}^{+}=(0, \infty)$ and $h(\infty)=\lim _{x \rightarrow \infty} h(x)$. Define

$$
r \stackrel{\circ}{=} h^{\prime}(0+) .
$$

By $\mathrm{A} 1$, this right hand limit exists in the extended sense, i.e., $0<r \leq \infty$.

Consider equation (3) when $\alpha=0$, i.e., consider the unforced, autonomous equation

$$
x_{t+1}=h\left(x_{t}\right) .
$$

If $r \leq 1$ assumption A1 implies $h(x)<x$ for $x>0$. In this case, solutions converge to the equilibrium 0 for all positive initial conditions $x_{0}>0$. In this case, we say 0 is globally attracting for $x_{0}>0$. On the other hand, if $r>1$ then there exists a unique positive root $x_{e}>0$ of the equation $x=h(x)$. In this case, solutions converge to the equilibrium $x_{e}$ for all positive initial conditions $x_{0}>0$, and we say $x_{e}$ is globally attracting for $x_{0}>0$.

Lemma 1 Assume A1. If $r \leq 1$, then the equilibrium 0 of (4) is globally attracting for $x_{0}>0$. If $r>1$ (including $r=\infty$ ) equation (4) has a globally attracting positive equilibrium $x_{e}$.

By a two-cycle we mean a solution $x_{t}$ of (3) whose even and odd iterates are both constant, i.e., $x_{2 k}=c_{0}$ and $x_{2 k+1}=c_{1}$ for all $t=0,1,2, \cdots$. We will denote a two-cycle by the ordered pair $\left(c_{0}, c_{1}\right)$. If both $c_{0}>0$ and $c_{1}>0$ then 
we say the two-cycle is positive. The solution of (3) converges to a two-cycle $\left(c_{0}, c_{1}\right)$ if

$$
\lim _{k \rightarrow \infty}\left(x_{2 k}, x_{2 k+1}\right)=\left(c_{0}, c_{1}\right) .
$$

A two-cycle $\left(c_{0}, c_{1}\right)$ is globally attracting for $x_{0}>0$ if solutions of (3) converge to $\left(c_{0}, c_{1}\right)$ for all initial conditions $x_{0}>0$.

In Section 2 we consider the asymptotic dynamics of the periodically forced equation (3). Theorem 2 establishes, when $r>1$, the existence of a (unique) positive two-cycle that is globally attracting for $x_{0}>0$. Our main result appears in Section 3. Theorem 3 shows, under certain concavity conditions, that the globally attracting two-cycle $\left(c_{0}, c_{1}\right)$ when $r>1$ is attenuant, i.e., it has a suppressed average in the sense that

$$
\frac{1}{2}\left(c_{0}+c_{1}\right)<x_{e} .
$$

This means the presence of periodic forcing in equation (3), i.e., $\alpha>0$, results in a global attractor with decreased average.

We will have need of the following two assumptions.

$$
\begin{array}{ll}
\text { A2: } & f \in C^{0}\left(R^{+}, R^{+}\right) \cap C^{1}\left(R_{0}^{+}, R^{+}\right) \\
& f^{\prime}(x)<0 \text { for all } x \in R_{0}^{+} \\
\text {A3: } & f \in C^{0}\left(R^{+}, R^{+}\right) \cap C^{2}\left(R_{0}^{+}, R^{+}\right) \\
& f^{\prime \prime}(x)>0 \text { for all } x \in R_{0}^{+}
\end{array}
$$

Note A1 and A3 together imply A2.

\section{Two-cycle Solutions}

A1 implies the solution of the periodically forced equation (3) with $x_{0}>0$ is positive, i.e., $x_{t}>0$ for all $t=0,1,2, \cdots$. Our goal in this section is determine when it is true that for all $x_{0}>0$ the solution tends to a unique positive twocycle solution. The components of a two-cycle $\left(c_{0}, c_{1}\right)$ necessarily satisfy the pair of "two-cycle" equations

$$
\begin{aligned}
& c_{1}=f\left(\frac{c_{0}}{1+\alpha}\right) c_{0} \\
& c_{0}=f\left(\frac{c_{1}}{1-\alpha}\right) c_{1} .
\end{aligned}
$$

Conversely, positive solutions $c_{0}>0, c_{1}>0$ of these simultaneous equations define a positive two-cycle $\left(c_{0}, c_{1}\right)$.

Theorem 2 Assume A1 and consider the periodically forced equation (3) for $\alpha>0$. If $r \leq 1$ then the equilibrium 0 is globally attracting for $x_{0}>0$. If $r>1$ (including $r=\infty$ ) then there exists a positive two-cycle $\left(c_{0}, c_{1}\right)$ that is globally attracting for $x_{0}>0$. If $f(x)$ is increasing (e.g., if A2 holds), then this two-cycle is strict, that is to say $c_{0} \neq c$. . 
Proof. Assume $x_{0}>0$. From the first composite of equation (3) we see that the even and odd iterates, $x_{2 k}$ and $x_{2 k+1}$, of the solution $x_{t}$ with initial condition $x_{0}>0$ satisfy the equations

$$
\begin{array}{ll}
e_{k+1}=E\left(e_{k}\right), & e_{0}=x_{0}>0 \\
o_{k+1}=O\left(o_{k}\right), & e_{1}=x_{1}>0
\end{array}
$$

respectively, where

$$
\begin{aligned}
& E(x) \stackrel{\circ}{=}\left(\frac{1}{1-\alpha} f\left(\frac{x}{1+\alpha}\right) x\right) f\left(\frac{x}{1+\alpha}\right) x \\
& O(x) \stackrel{\circ}{=}\left(\frac{1}{1+\alpha} f\left(\frac{x}{1-\alpha}\right) x\right) f\left(\frac{x}{1-\alpha}\right) x
\end{aligned}
$$

Here $e_{k}=x_{2 k}$ and $o_{k}=e_{2 k+1}$ for $k=0,1,2, \cdots$. Alternatively we can write

$$
\begin{aligned}
& E(x)=(1-\alpha) h\left(\frac{1+\alpha}{1-\alpha} h\left(\frac{x}{1+\alpha}\right)\right) \\
& O(x)=(1+\alpha) h\left(\frac{1-\alpha}{1+\alpha} h\left(\frac{x}{1-\alpha}\right)\right) .
\end{aligned}
$$

Assumption A1 implies $E(x)$ and $O(x)$ also satisfy A1. A calculation shows

$$
E^{\prime}(0+)=O^{\prime}(0+)=r^{2} .
$$

From Lemma 1 applied to both equations in (7) we conclude that both sequences, and hence $x_{t}$, converge to 0 if $r \leq 1$.

If, on the other hand, $r>1$ then each equation in (7) has a globally attracting, positive equilibrium. Thus, the even iterates $e_{k}=x_{2 k}$ converge to the unique positive root $c_{0}>0$ of $x=E(x)$ and the odd iterates $o_{k}=e_{2 k+1}$ converge to the unique positive root $c_{1}>0$ of $x=O(x)$. If we let $t=2 k$ in equation (3) and let $k \rightarrow \infty$, then we find by continuity that $c_{0}$ and $c_{1}$ satisfy the first of the two-cycle equations (6). Similarly, letting $t=2 k+1$ and passing $k \rightarrow \infty$ in (3) we find that $c_{0}$ and $c_{1}$ also satisfy the second equation in (6). Thus, the two constants $c_{0}$ and $c_{1}$ define a positive two-cycle $\left(c_{0}, c_{1}\right)$ to which $x_{t}$ converges.

If $c_{0}=c_{1}=c$ then from the two-cycle equations (6) it follows that

$$
f\left(\frac{c}{1+\alpha}\right)=f\left(\frac{c}{1-\alpha}\right) .
$$

If $f$ is increasing, we arrive at a contradiction. Thus, in this case, $c_{0} \neq c_{1}$ and the two-cycle is strict.

\section{Attenuant two-cycles}

For $r>1$ (including $r=\infty$ ) the autonomous equation (4) has a unique positive equilibrium $x_{e}>0$. Changing variables from $x$ to $x / x_{e}$ in equation (4), we 
can assume without loss in generality that the unique positive equilibrium is 1 . Under this assumption $h(x)$ satisfies the added restriction

$$
\text { A4: } \quad h(1)=1
$$

(and hence $f(1)=1$ ). Note that A1 and A4 together imply $r>1$. Thus, under these two assumptions Theorem 2 implies the existence of a positive two-cycle $\left(c_{0}, c_{1}\right)$ that is globally attracting for $x_{0}>0$. This two-cycle is attenuant if and only if

$$
\frac{1}{2}\left(c_{0}+c_{1}\right)<1 .
$$

Theorem 3 Assume A1, A3 and A4. For each $\alpha \in(0,1)$ the positive, globally attracting (for $x_{0}>0$ ), strict two-cycle of the periodically forced equation (3) is attenuant.

Before proving this theorem we establish some lemmas.

Lemma 4 Assume A1 and A2. For $r>1$ let $\left(c_{0}, c_{1}\right)$ be the positive two-cycle guaranteed by Theorem 2. Then $0<c_{0}<c_{1}$ for each $\alpha \in(0,1)$.

Proof. For purposes of contradiction assume $c_{1} \leq c_{0}$. From A2 and the second of the two-cycle equations (6) we have

$$
c_{0}=f\left(\frac{c_{1}}{1-\alpha}\right) c_{1}<f\left(\frac{c_{1}}{1+\alpha}\right) c_{1}=(1+\alpha) h\left(\frac{c_{1}}{1+\alpha}\right) .
$$

By A1 and the first of the two-cycle equations (6) we have

$$
\begin{aligned}
(1+\alpha) h\left(\frac{c_{1}}{1+\alpha}\right) & \leq(1+\alpha) h\left(\frac{c_{0}}{1+\alpha}\right) \\
& =f\left(\frac{c_{0}}{1+\alpha}\right) c_{0}=c_{1},
\end{aligned}
$$

which gives the contradiction $c_{0}<c_{1}$.

Lemma 5 Assume $A 1$ and A4. Then

$$
\frac{1}{2}\left[(1+\alpha) h\left(\frac{1}{1+\alpha}\right)+(1-\alpha) h\left(\frac{1}{1-\alpha}\right)\right]<1
$$

for all $\alpha \in(0,1)$.

Proof. For $\alpha \in[0,1)$ define the function

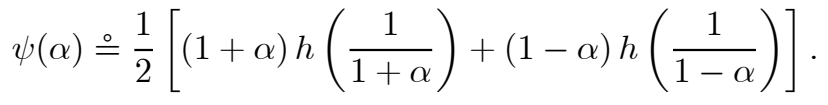


Since $\psi(0)=1$, it suffices to show $\psi(\alpha)$ is a decreasing function of $\alpha$. For $\alpha \in(0,1)$ a calculation shows

$$
\psi^{\prime}(\alpha)=\phi(1+\alpha)-\phi(1-\alpha)
$$

where

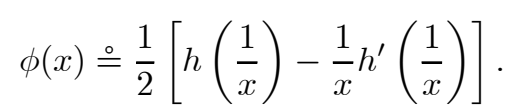

By the Mean Value Theorem, $\psi^{\prime}(\alpha)=2 \alpha \phi^{\prime}(c)$ for some $c \in(1-\alpha, 1+\alpha)$. However, by A1

$$
\phi^{\prime}(c)=\frac{1}{2 c^{3}} h^{\prime \prime}\left(\frac{1}{c}\right)<0
$$

and hence $\psi^{\prime}(\alpha)<0$.

Lemma 6 Assume A1, A3 and A4. Then $h^{\prime}\left(\frac{1}{1+\alpha}\right)<1$ for all $\alpha \in[0,1)$.

Proof. Since $h^{\prime \prime}(x)<0$ for all $x>0$, it suffices to show $h^{\prime}\left(\frac{1}{2}\right)<1$. By the Mean Value Theorem, $1-f\left(\frac{1}{2}\right)=f(1)-f\left(\frac{1}{2}\right)=\frac{1}{2} f^{\prime}(c)$ for some $c \in\left(\frac{1}{2}, 1\right)$. However, A3 implies $f^{\prime}(c)>f^{\prime}\left(\frac{1}{2}\right)$, and thus $1>\frac{1}{2} f^{\prime}\left(\frac{1}{2}\right)+f\left(\frac{1}{2}\right)=h^{\prime}\left(\frac{1}{2}\right)$.

Proof of Theorem 3. If $c_{1} \leq 1$, the two-cycle is attenuant by Lemma 4 . Therefore we assume $c_{1}>1$. We can rewrite the two-cycle equations (6) as

$$
\begin{aligned}
& c_{1}=(1+\alpha) h\left(\frac{c_{0}}{1+\alpha}\right) \\
& c_{0}=(1-\alpha) h\left(\frac{c_{1}}{1-\alpha}\right) .
\end{aligned}
$$

Consider the second order Taylor expansions at $c=1$

$$
(1 \pm \alpha) h\left(\frac{c}{1 \pm \alpha}\right)=(1 \pm \alpha) h\left(\frac{1}{1 \pm \alpha}\right)+h^{\prime}\left(\frac{1}{1 \pm \alpha}\right)(c-1)+H_{ \pm}(c, \alpha)
$$

where

$$
H_{ \pm}(c, \alpha) \stackrel{\circ}{=}\left(\frac{1}{1 \pm \alpha}\right) h^{\prime \prime}\left(\xi_{ \pm}\right)(c-1)^{2}
$$

and $\xi_{ \pm}$is a nonzero number between 1 and $c$. By A1, $H_{ \pm}(c, \alpha) \leq 0$. The twocycle equations become

$$
\begin{aligned}
& \text { (a) } c_{1}=(1+\alpha) h\left(\frac{1}{1+\alpha}\right)+h^{\prime}\left(\frac{1}{1+\alpha}\right)\left(c_{0}-1\right)+H_{+}\left(c_{0}, \alpha\right) \\
& \text { (b) } c_{0}=(1-\alpha) h\left(\frac{1}{1-\alpha}\right)+h^{\prime}\left(\frac{1}{1-\alpha}\right)\left(c_{1}-1\right)+H_{-}\left(c_{1}, \alpha\right) .
\end{aligned}
$$


Note

$$
h^{\prime}\left(\frac{1}{1-\alpha}\right)\left(c_{1}-1\right)<h^{\prime}\left(\frac{1}{1+\alpha}\right)\left(c_{1}-1\right)
$$

since $h^{\prime \prime}(x)<0$ for $x>0$. Thus, from (9b) we have

$$
c_{0}<(1-\alpha) h\left(\frac{1}{1-\alpha}\right)+h^{\prime}\left(\frac{1}{1+\alpha}\right)\left(c_{1}-1\right)
$$

If we add (9a) to both sides of this inequality, we obtain

$$
\begin{aligned}
\frac{1}{2}\left(c_{0}+c_{1}\right)< & \frac{1}{2}\left[(1+\alpha) h\left(\frac{1}{1+\alpha}\right)+(1-\alpha) h\left(\frac{1}{1-\alpha}\right)\right] \\
& +h^{\prime}\left(\frac{1}{1+\alpha}\right)\left(\frac{1}{2}\left(c_{0}+c_{1}\right)-1\right)
\end{aligned}
$$

Lemma 5 implies

$$
\frac{1}{2}\left(c_{0}+c_{1}\right)<1+h^{\prime}\left(\frac{1}{1+\alpha}\right)\left(\frac{1}{2}\left(c_{0}+c_{1}\right)-1\right)
$$

and hence

$$
\left(\frac{1}{2}\left(c_{0}+c_{1}\right)-1\right)\left(1-h^{\prime}\left(\frac{1}{1+\alpha}\right)\right)<0 .
$$

By Lemma 6 the second factor is positive and therefore the first factor is negative.

By inequality (8), it is certainly true that a two-cycle $\left(c_{0}, c_{1}\right)$ is attenuant if $\max \left\{c_{0}, c_{1}\right\} \leq 1$. In this case, we call the two-cycle strongly attenuant. If, on the other hand, an attenuant two-cycle satisfies $\max \left\{c_{0}, c_{1}\right\}>1$ we call the two-cycle weakly attenuant.

Theorem 7 Assume A1, A3 and A4. For $\alpha \in(0,1)$ sufficiently close to 0 the positive, globally attracting (for $x_{0}>0$ ), strict two-cycle $\left(c_{0}, c_{1}\right)$ of the periodically forced equation (3) is weakly attenuant. For $\alpha \in(0,1)$ sufficiently close to 1 the two-cycle is strongly attenuant.

Proof. From the two-cycle equations (6) we have the equation

$$
c_{1}=f\left(\frac{1}{1+\alpha} f\left(\frac{c_{1}}{1-\alpha}\right) c_{1}\right) f\left(\frac{c_{1}}{1-\alpha}\right) c_{1}
$$

and hence

$$
1=f\left(\frac{1}{1+\alpha} f\left(\frac{c_{1}}{1-\alpha}\right) c_{1}\right) f\left(\frac{c_{1}}{1-\alpha}\right)
$$

for $c_{1}>0$. By A1 and A2, the right hand side, which is identical to

$$
f\left(\frac{1-\alpha}{1+\alpha} h\left(\frac{c_{1}}{1-\alpha}\right)\right) f\left(\frac{c_{1}}{1-\alpha}\right),
$$


is a decreasing function of $c_{1}>0$. Therefore, any positive root of (11) is unique. In particular the root $c_{1}=1$ when $\alpha=0$ unique. The implicit function theorem implies there exists a (locally unique), continuously differentiable root $c_{1}=$ $c_{1}(\alpha)$ for $\alpha$ near 0 satisfying $c_{1}(0)=1$.

Differentiating both sides of equation (11) with respect to $\alpha$ and evaluating the result at $\alpha=0$ we obtain

$$
c_{1}^{\prime}(0)=-\frac{f^{\prime}(1)}{2+f^{\prime}(1)} \text {. }
$$

From $f^{\prime}(1)<0$ and $0<h^{\prime}(1)=1+f^{\prime}(1)$ it follows that $c_{1}^{\prime}(0)>0$. Thus, $c_{1}(\alpha)>1$ for $\alpha>0$ sufficiently small and the two-cycle is weakly attenuant.

From equation (10) we derive the inequalities

$$
\begin{aligned}
0 & <c_{1}=(1+\alpha) f\left(\frac{1-\alpha}{1+\alpha} f\left(\frac{c_{1}}{1-\alpha}\right) \frac{c_{1}}{1-\alpha}\right) \frac{1-\alpha}{1+\alpha} f\left(\frac{c_{1}}{1-\alpha}\right) \frac{c_{1}}{1-\alpha} \\
& =(1+\alpha) h\left(\frac{1-\alpha}{1+\alpha} h\left(\frac{c_{1}}{1-\alpha}\right)\right) \\
& \leq(1+\alpha) h\left(\frac{1-\alpha}{1+\alpha} h(\infty)\right)
\end{aligned}
$$

valid for all $\alpha \in(0,1)$. This implies

$$
\lim _{\alpha \rightarrow 1-} c_{1}(\alpha)=0
$$

which implies $c_{1}<1$ for $\alpha$ near 1 . By Lemma $4, \max \left\{c_{0}, c_{1}\right\}<1$ and the two-cycle is strongly attenuant for $\alpha$ near 1 .

\section{Examples}

Consider the periodically forced Beverton-Holt equation (2). For this equation

$$
f(x)=r \frac{1}{1+(r-1) x}, \quad h(x)=r \frac{1}{1+(r-1) x} x, \quad r>1 .
$$

All assumptions A1, A3 and A4 (and hence A2) are satisfied. Therefore, for each $\alpha \in(0,1)$ the equation (2) has an attenuant, positive, strict two-cycle $\left(c_{0}, c_{1}\right)$ that is globally attracting for $x_{0}>0$. In fact, in this example the two-cycle is $\left(c_{0}, c_{1}\right)$ where

$$
c_{0}=\frac{(r+1)\left(1-\alpha^{2}\right)}{1-\alpha+r(1+\alpha)}, \quad c_{1}=\frac{(r+1)\left(1-\alpha^{2}\right)}{1+\alpha+r(1-\alpha)}
$$

and

$$
\frac{1}{2}\left(c_{0}+c_{1}\right)=\frac{1+2 r+r^{2}}{1+2\left(\frac{1+\alpha}{1-\alpha}\right) r+r^{2}}<1 .
$$

The cycle is weakly attenuant $\left(c_{1}>1\right)$ for $0<\alpha<\frac{r-1}{r+1}$ and strongly attenuant for $\frac{r-1}{r+1} \leq \alpha<1$. See Figure 1 for an example. 


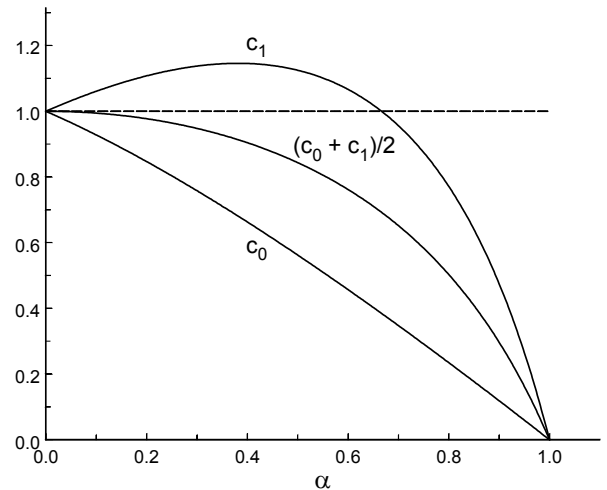

FIGURE 1. The two-cycles of the periodically forced Beverton-Holt equation (2) and their averages are plotted as functions of the relative amplitude $\alpha$ when $r=5>1$.

Other examples to which Theorems 2, 3 and 7 apply are

$$
f(x)=r \frac{1}{1+(r-1) x^{p}} \text { for } 0<p<1, r>1
$$

and

$$
f(x)=x^{p-1} \text { for } 0<p<1 .
$$

The latter example is a case when $r=\infty$. Figure 2 illustrates this example when $p=1 / 2$.

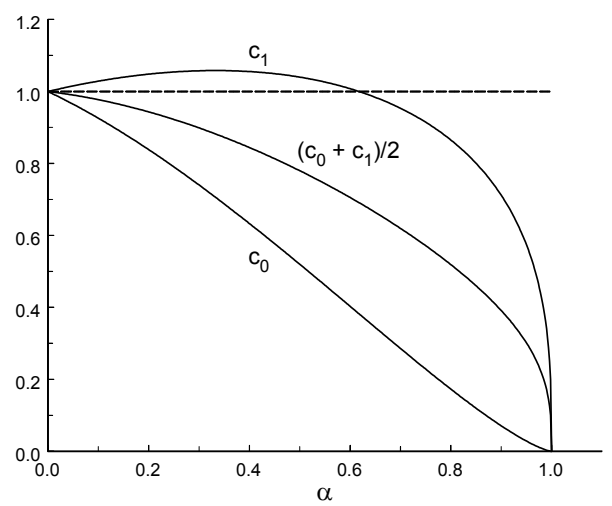

FIGURE 2. The two-cycles of the periodically forced equation (3), with $f(x)=x^{-1 / 2}$, and their averages are plotted as functions of the relative amplitude $\alpha$. In this case $r=\infty$. 


\section{References}

[1] B. D. Coleman, On the growth of populations with narrow spread in reproductive age. I. General theory and examples, J. Math. Biol. 6 (1978), $1-19$

[2] C. S. Coleman and J. C. Frauenthal, Satiable egg eating predators, Math. Biosci. 63 (1983), 99-119

[3] R. F. Costantino, J. M. Cushing, B. Dennis, R. A. Desharnais, S. M. Henson, Resonant population cycles in temporally fluctuating habitats, Bull. Math. Biology 60, No. 2 (1998), 247-275

[4] J. M. Cushing, Oscillatory population growth in periodic environments, Theor. Pop. Biol. 30 (1987), 289-308

[5] J. M. Cushing, Periodically forced nonlinear systems of difference equations, J. Diff. Eq. Appl. 3 (1998), 547-561

[6] S. M. Henson, Existence and stability of nontrivial periodic solutions of periodically forced discrete dynamical systems. J. Diff Eq. Appl. 2 (1996), $315-331$

[7] S. M. Henson, The effect of periodicity in maps, J. Diff Eq. Appl. 5 (1999), $31-56$

[8] S. M. Henson, Multiple attractors and resonance in periodically forced population models, Physica D 140 (2000), 33-49

[9] S. M. Henson and J. M. Cushing, The effect of periodic habitat fluctuations on a nonlinear insect population model, J. Math. Biol. 36 (1997), 201-226

[10] R. M. May, Stability and complexity in model ecosystems, Princeton University Press, Princeton, New Jersey, 1973

[11] R. M. Nisbet and W. S. C. Gurney, Modelling Fluctuating Populations, Wiley \& Sons, New York, 1982

[12] S. Rosenblat, Population models in a periodically fluctuating environment, J. Math. Biol. 9 (1980), 23-36 